\title{
Feasibility of laser targeted photo-occlusion of ocular vessels
}

\author{
Sanjay Asrani, Ran Zeimer
}

\begin{abstract}
Aims/Background-Neovascularisation occurs in many major ocular diseases such as diabetes, age-related macular degeneration, and sickle cell disease. Laser photocoagulation is typically used to obliterate the vessels but it also causes severe damage to adjacent normal tissues. This is a very significant limitation especially in the treatment of choroidal neovascularisation which often covers large areas of the posterior pole and the fovea. A method, laser targeted delivery, has been developed capable of releasing drugs locally and non-invasively in the choroidal or retinal vasculature. This method could be used to target a photosensitiser to neovascular membranes and cause their selective occlusion by irradiating them. The targeting properties of the method promise to yield a treatment for neovascularisation that does not damage adjacent tissues and thus preserves vision. The purpose of the present study was to test the feasibility of occluding ocular vessels with this method.
\end{abstract}

Method-The iris vessels of the albino rat were chosen because the treatment could be assessed unequivocally and followed with time. Aluminium phthalocyanine tetrasulphonate was encapsulated in heat sensitive liposomes and administered systemically. The iris vessels were irradiated with a yellow laser to raise their temperature to $41^{\circ} \mathrm{C}$, cause a phase transition in the liposomes and thereby locally release the photosensitiser. The laser was also used to excite the released photosensitiser and cause occlusion. The effect was monitored immediately and for 8 months thereafter. Controls for the effect of the laser and the unencapsulated drug were conducted.

Results-The results demonstrated that occlusion can be achieved and sustained for the period of follow up. The controls showed that the effect was not due to heat or to the activation of the low dose of free drug.

Conclusion-These preliminary findings indicate that laser targeted photo-occlusion is a promising new method for the treatment of neovascularisation.

(Br f Ophthalmol 1995; 79: 766-770)

Age-related macular degeneration (ARMD) is one of the leading causes of severe vision loss in people over the age of $50 . .^{1-3}$ As the population ages, a greater number of elderly persons will become blind from ARMD than from glaucoma and diabetic retinopathy combined. ${ }^{4}$ Choroidal neovascularisation (CNV), common in ARMD, is usually treated by intense laser photocoagulation. However, the thermal damage and the scarring of large macular areas cause a dramatic loss of vision (typically to $20 / 200$ or worse) when the fovea is treated. ${ }^{5}$ The treatment is performed, none the less, in cases in which it has been shown to prevent progressive visual loss. These eligible cases, only $25 \%$ of the eyes with CNV, have a well defined ('classic') CNV of limited size." The remaining $75 \%$ of the eyes are left untreated because no useful vision would be spared. Additionally, in a majority of the patients $(54 \%)$ treated by laser photocoagulation, the new blood vessels recur thereby requiring further treatment. ${ }^{6}$ The recurrence has been attributed to incomplete identification of the CNV and also to damage to normal tissues, scar formation, and breaks in Bruch's membrane, all of which are believed to elicit growth of new vessels.

A more efficient treatment could consist of occluding the new vessels by pharmacologically damaging the cells lining their lumen. Such damage would cause aggregation of blood products and long lasting occlusions. Photodynamic therapy has raised interest as such a potential method. It consists of injecting a photosensitive agent that, when exposed to the appropriate wavelength of light, generates free radicals and singlet oxygen. These agents then attack the blood vessel wall and cause occlusion. The success of photodynamic therapy in causing regression of tumours has been attributed mostly to vessel occlusion. ${ }^{7}$ Thus, it is not surprising that photodynamic therapy, following systemic injection of the agent, has been applied to the eye in an attempt to occlude vessels. ${ }^{8-10}$ These studies showed that occlusion can be achieved by chemical means without causing any thermal damage. However, besides being present in the $\mathrm{CNV}$ the photosensitiser also fills the retinal and choroidal vasculatures and leaks into the retinal tissue. Consequently, upon irradiation, damage to the retinal photoreceptors, the outer retinal layers and, potentially, to the retinal capillaries is anticipated. Such damage was observed in the work of Kliman et al. ${ }^{9} 10$ There is thus a need for a method of occluding neovascularisation which does not cause a scar and promises to spare the overlying retina and the adjacent choriocapillaris. Such a treatment would save sight in a large portion of the population and would lead to significant savings to society.

We have developed a method of selective drug delivery that can be applied to occlude 
new blood vessels. The method, laser targeted delivery (LTD), consists of encapsulating a drug in heat sensitive liposomes, injecting them intravenously, and releasing their content at the site of choice by non-invasively warming up the targeted tissue (retina or choroid) momentarily with a laser pulse directed through the pupil of the eye. ${ }^{11}$ The temperature needed for the phase transition and drug release is $41^{\circ} \mathrm{C}$ and thus within a safe range. LTD has been successfully applied to deliver locally a dye specifically in the retinal ${ }^{12-14}$ and choroidal vasculature. ${ }^{15}$ This has yielded angiograms of retinal and choroidal vasculatures with a quality that cannot be obtained by conventional angiography. ${ }^{13} 15$ The results have illustrated that, by exploiting the optical and haemodynamic properties at the posterior pole, one can deliver a substance in the subretinal vasculature without releasing it from the liposomes that circulate in the retinal vessels; and, conversely, one can target the retinal vasculature while minimising the release in the choroid. This indicates that the targeting is not only local but that it can also be selective in superimposed tissues. Moreover, the dose can be controlled by repeating the procedure as long as the liposomes circulate in the blood. In our experience, this time period is at least 1 hour. ${ }^{16}$

Laser targeted photo-occlusion, which is the combination of laser targeted delivery and photodynamic therapy, has the potential of solving many of the limitations of systemic photodynamic therapy as well as those of laser photocoagulation. The method is based on encapsulating a photosensitiser in the heat sensitive liposomes, releasing it in the $\mathrm{CNV}$, and immediately activating it to cause local damage.

Photodynamic therapy for malignant tumours is usually done at 24 hours after the administration of the agent. ${ }^{17}$ The shortest time interval between agent injection and subsequent irradiation reported so far has been 2 minutes. ${ }^{18}$ The combination of LTD and photodynamic therapy would require that the photosensitiser cause its effects during its presence in the lumen for a duration of a few seconds or less. The present study was thus aimed at evaluating the feasibility of laser targeted photo-occlusion by testing whether release of the photosensitiser with simultaneous irradiation with light produces occlusion.

\section{Materials and method}

Aluminium phthalocyanine tetrasulphonate (AlPcS4) was considered to be an optimal photosensitiser because its peak absorption, around $675 \mathrm{~nm}$, assures penetration through blood (only $11 \%$ absorption by a $100 \mu \mathrm{m}$ layer); it is water soluble and can be encapsulated efficiently; it has one of the highest absorption coefficients (30 times higher than that of haematoporphyrin derivative ${ }^{19}$ ) ensuring high sensitisation with minimal amount of light on the retina; it is a well defined compound which can be synthesised with high purity; and it is removed within 24 hours from the blood ${ }^{20}$ thereby reducing the period of light sensitivity which has been a limitation of other photosensitisers. ${ }^{21}$ This photosensitiser has been demonstrated to be non-toxic in a number of species ${ }^{20}$ and no side effects have been reported when it was used intraperitoneally in few patients. ${ }^{22}$

AlPcS4 (Porphyrin Products, Logan, UT, USA) was stored at $-20^{\circ} \mathrm{C}$ in the dark in powder form. It was dissolved in sterile water for injection and filtered through a $0.2 \mu \mathrm{m}$ syringe filter.

Dipalmitoylphosphatidylcholine and dipalmitoylphosphatidylglycerol were obtained from Avanti Polar Lipids (Pelham, AL, USA) and used without further purification. Liposomes were prepared by reverse phase evaporation following a method described in detail previously. ${ }^{11} 12$ AlPcS4 dissolved in sterile water was encapsulated in the liposomes to yield a concentration of $5 \mathrm{mg} / \mathrm{ml}$ of formulation.

Quality control tests were developed to ensure safety of the animals and the success of the experiments. The raw materials were tested to ensure their sterility and the glassware was autoclaved. Liposomes were prepared using aseptic techniques and tested for sterility by incubating them at $37^{\circ} \mathrm{C}$ on blood agar plates and in thioglycollate media for 72 hours. The limulus amoebocyte lysate (LAL) test was used to ensure that the liposomes and water were free from pyrogens, especially endotoxins. Since the size distribution of the liposomes affects their half life in the blood stream and liposomes larger than $0.45 \mu \mathrm{m}$ could cause side effects, the liposome preparation was filtered through a $0.4 \mu \mathrm{m}$ polycarbonate filter. To test whether laser targeted photo-occlusion can cause occlusion of vessels, it was imperative to create a model that could yield an unequivocal answer as to its efficacy. The iris vessels of the albino rat were considered the model of choice, because they are readily visible and untreated portions could serve as well defined and stable controls. The protocol was designed in accordance with the ARVO Resolution on the Use of Animals in Research and approval was obtained from the Institutional Review Board. The experiments were conducted on six albino male Sprague Dawley rats weighing 250-300 g. They were anaesthetised with ketamine $(50 \mathrm{mg} / \mathrm{kg})$ and xylazine $(10 \mathrm{mg} / \mathrm{kg})$ intramuscularly.

Ideally, the irradiation of the photosensitiser should be performed at the maximal absorption of $675 \mathrm{~nm}$ to cause optimal photosensitisation and the release from the liposomes should be achieved by a laser at a wavelength strongly absorbed by blood (the only pigment in the iris of the albino rat). But, since we did not have access to two lasers operating simultaneously, the same laser was used for both purposes. A dye laser (Coherent, Palo Alto, CA, USA), set at $577 \mathrm{~nm}$, was chosen to match the high absorption of blood at this wavelength ${ }^{23}$ thereby raising the temperature of the iris vessels efficiently. On the other hand, at this wavelength, the absorption of AlPcS4 is 14 times less than at the peak. 


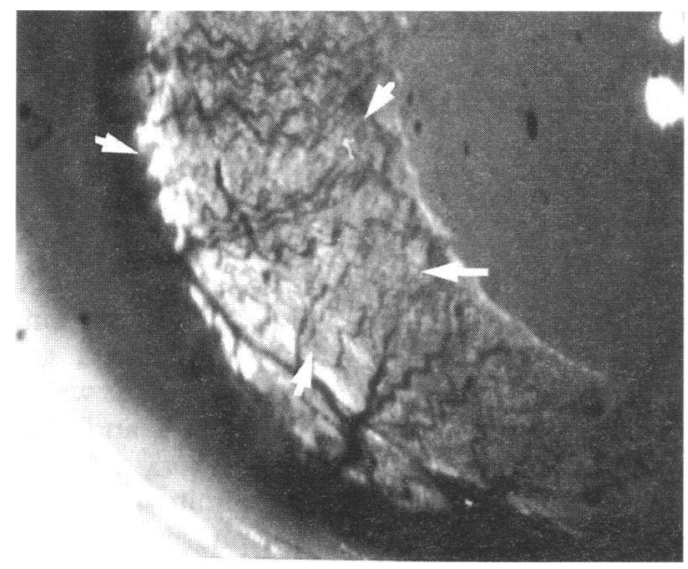

Figure 1 Iris of an albino rat before laser targeted photo-occlusion. Red-free video image of the iris before treatment. The arrows delineate the area designated for treatment.

The laser was coupled to a slit-lamp biomicroscope and was part of a clinical laser delivery system.

The power of the laser was set according to the calculations of Bebie and coworkers for the power necessary to warm blood vessels embedded in a transparent medium by $4^{\circ} \mathrm{C} .^{24}$ This temperature rise is sufficient to release the contents of the heat sensitive liposomes. The power of the laser spot was set to $50 \mathrm{~mW}$ and $400 \mu \mathrm{m}$ diameter.

Before the injection, the irides of both eyes were imaged to obtain a baseline. The liposome preparation was injected intravenously to yield a dose of AlPcS4 of $7.5 \mathrm{mg} / \mathrm{kg}$. A portion of the iris, sustaining an angle of approximately $60^{\circ}$, was treated. The number of pulses necessary to obtain an effect was determined in pilot experiments. The remaining animals were then treated with these variables. To control for the effect of the laser by itself, a similar portion of the left iris was exposed to the laser before the injection of the photosensitiser-liposome preparation. The preparation was then injected and the right iris was treated within 5 minutes of injection.

The heat sensitive liposomes may release some of their content (10 to $15 \%$ ) at body temperature. ${ }^{11}$ To assess the effect of this unencapsulated portion of the dose a second control was performed. The same liposome preparation was lysed by heating it beyond the phase transition and $20 \%$ of the dose of the lysed preparation was administered to another rat. The iris was then treated within 5 minutes of the injection with the same laser variables as above. The iris was visualised continuously for 15 minutes to detect any change in the vessels. To control for potential individual variability the treatment was followed 20 minutes later by an injection, to the same animal, of intact liposomes and a different quadrant was treated with the same laser delivery protocol.

The eyes were followed up by obtaining redfree video images with a video camera (Texas Instruments, Dallas, TX, USA) coupled to one of the viewing arms of the slit-lamp biomicroscope. The video output was recorded on magnetic tape with a high frequency videorecorder

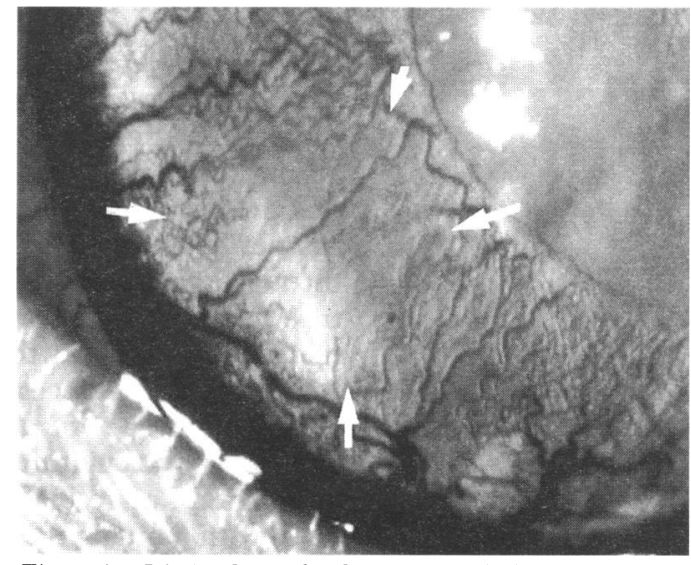

Figure 2 Iris 25 days after laser targeted photo-occlusion. Note the complete occlusion within the area treated (marked by the arrows). The vessel at the centre of the treated area remained open immediately after the procedure and was apparently undertreated. 'Ghost vessels' are visible and indicate that the lack of visible patent vessels is not the result of tissue opacity.

(Sony, Tokyo, Japan) and later digitised with a frame grabber (Epix, Northbrook, IL, USA).

\section{Results}

Pilot trial in four animals established that 40 pulses of 0.5 second yielded visible results. These variables were adopted for the rest of the study.

When the right eye was treated after liposomes injections, vessel engorgement followed by vessel spasms, haemorrhages, and local iris tissue expansion were observed upon the delivery of 20 pulses. Local iris tissue constriction occurred at the end of 40 pulses. In contrast, in the left control eye (treated before liposome injection), no effect was noticed in the blood vessels or the iris tissue during the delivery.

The follow up examinations with redfree imaging revealed, in the treated eye, haemorrhages which cleared within a week, leaving the iris tissue with non-perfused vessels. The results at 25 days are illustrated in Figure 2 and compared with the iris before treatment shown in Figure 1. All the vessels in the treated area were occluded and most of them could be seen as non-perfused vessels (ghost vessels). The remaining vessel at the centre was patent. It had, however, been patent immediately after the treatment and throughout the follow up. During the follow up period, which was done monthly for 8 months, no reperfusion of occluded vessels was observed. In contrast, the follow up of the control eye did not reveal any ophthalmoscopically visible pathology at any time point.

In the second experiment, after the injection of the free photosensitiser at $20 \%$ of the encapsulated dose, no response was observed in the blood vessels or the pupil up to 15 minutes after completion of 40 deliveries. When the intact liposome preparation was injected in the same animal and another quadrant was treated, the occlusive events mentioned were observed after the first 20 pulses. 


\section{Discussion}

The results demonstrate the feasibility of laser targeted photo-occlusion. The control experiments did not yield the effects which were observed when the photosensitiser was released at high concentration for the liposomes and simultaneously irradiated. This assures that the occlusion was not due to the direct effect of the laser on the tissue or to the activation of the low dose of free photosensitiser. In contrast, the treatment caused occlusion which lasted for the long term follow up. The fact that ghost vessels were visible from the seventh day onwards provides conclusive evidence that the observation of lack of perfused vessels was the result of occlusion and not artefacts such as overlying oedematous fluid or exudate. The single vessel that is seen patent in the figure was apparently not covered adequately by the raster of spots during treatment.

These results reveal, for the first time, that photosensitisers can yield occlusive effects when activated during their presence inside the blood stream. It is unlikely that, owing to the short passage of the bolus, the activated photosensitiser had sufficient time to exit the lumen. This sheds some light on one of the mechanisms of photodynamic therapy as it indicates that the penetration of the photosensitiser into the tissue or the cells is not a prerequisite for its effect.

But, most importantly, this study illustrates the feasibility of causing occlusion with our method of laser targeted delivery. The success in occluding vessels in the iris is encouraging because these are normal and subjected to a high pressure head. Abnormal new vessels such as those encountered in age-related macular degeneration are likely to be more prone to damage owing to their defective endothelial lining. On the other hand, since some of the photosensitiser (presently approximately $15 \%$ ) exits the liposomes at body temperature, it will be necessary to elucidate if the leakage of some of this non-encapsulated dye into the retina will be sufficient to cause tissue damage when exposed to irradiation.

The lack of visible histological damage after laser exposure necessary for the release of liposomes in the choroid has been demonstrated. ${ }^{15}$ This is not surprising as the exposure meets the American National Standard for the Safe Use of Lasers. ${ }^{25}$ The safety of the exposure needed for the excitation of the photosensitiser can be extrapolated from this study. With a laser at $675 \mathrm{~nm}, \mathrm{AlPcS} 4$ can be excited with 14 times less light than with the present laser operating at $577 \mathrm{~nm} .{ }^{19}$ Thus an energy of 40 pulses at $50 \mathrm{~mW}$ for $0.5 \mathrm{~s} / 14=72 \mathrm{~mJ}$ will be sufficient. According to the recent American National Standard for the Safe Use of Lasers, ${ }^{25}$ which includes an additional safety factor for pharmacologically dilated pupils and immobilised globes, a power of $22 \mathrm{~mW}$ on a $400 \mu \mathrm{m}$ spot is safe for $0.7 \mathrm{~s}$. For repetitive pulses, the standards mandate that the power be reduced further by $n^{1 / 4}$. Thus, if eight pulses are delivered, the power needs to be reduced to $13 \mathrm{~mW}$. But eight such pulses of $0.7 \mathrm{~s}$ duration suffice to yield the necessary energy of $72 \mathrm{~mJ}$ while remaining within the permissible range for safe exposure.

No significant toxicity can be anticipated from the systemic administration of liposomes. The lipids used in the preparation, namely dipalmitoylphosphatidyl choline (DPPC) and dipalmitoylphosphatidyl glycerol (DPPG) are among the least toxic lipids ${ }^{26}$ used for the preparation of liposomes. Both DPPC and DPPG as well as preparations of large unilamellar liposomes have been used successfully in human clinical trials. ${ }^{27-30} \mathrm{~A}$ recent overview of the toxicology of liposomes in humans concluded that the observed adverse effects were mostly characteristic of the encapsulated drug. ${ }^{31}$ Nevertheless, a thorough toxicology study of our specific preparation will be necessary before application in humans.

LTD shares the basic advantages of other systemic liposomal delivery systems which protect most organs from exposure to the agent thereby reducing the toxicity. Phthalocyanines have been administered safely at high doses in more than one animal species ${ }^{20}$ and were found to have minimal skin phototoxicity. ${ }^{21}$

Laser targeted photo-occlusion has thus the potential of providing a therapy that can surpass laser photocoagulation and conventional photodynamic therapy for the following reasons:

(1) Our results of the specific visualisation of the choroidal vasculature ${ }^{15}$ indicate that the photosensitiser can be released in the subretinal vasculature while avoiding release in the retinal capillaries. Thus these vessels will not be damaged during the irradiation.

(2) By irradiating immediately after the release, the damage can be limited to the vessels perfused by the short bolus. Accumulation of photosensitiser in the interstitial tissues and subsequent damage upon irradiation can be avoided.

(3) There are clear indications that CNVs are perfused by a slower flow than the normal choriocapillaris. ${ }^{32}$ The photosensitiser could be released and the tissue irradiated only after enough time has elapsed to ensure clearance from the normal choriocapillaris. This would ensure preservation of the choriocapillaris which is crucial to the maintenance of the retinal pigment epithelium.

(4) The non-thermal occlusion may avoid extensive scarring and breaks in Bruch's membrane both believed to increase the risk of neovascularisation recurrence.

Laser targeted photo-occlusion thus holds promise to benefit a large portion of the population with macular degeneration by providing selective occlusion with better preservation of vision. The absence of iatrogenic damage could provide treatment of large lesions and occult CNV by covering suspected areas as well.

Supported in part by research grant EY 07768 from the National Institutes of Health, Bethesda, Maryland, USA.

Dr Zeimer holds a patent on the technology.

Xiao Yun Zhang, MD, prepared the liposomes.

1 Leibowitz HM, Krueger DE, Maunder LR. The Framingham eye study monograph; An ophthalmological and epidemiological study of cataract, glaucoma, diabetic retinopathy, macular degeneration, and visual acuity in a general population of 2631 adults, 1973-1975. Surv Ophthalmol 1980; 24 (suppl): 335-610. 
2 Sorsby A. The incidence and causes of blindness in England and Wales 1963-1968. In: Ministry of Health Reports on Public Health and Medical Subjects. 128th ed. London: HMSO, 1972 .

3 Ferris FL. Senile macular degeneration: review of epidemiologic features. Am $\mathcal{f}$ Epidemiol 1983; 118: 132-51.

4 National Advisory Eye Council. Report of the retinal diseases panel. In: Vision research a national plan diseases panel. In: Vision research a national plan and Human Services, 1994 .

5 Bressler NM, Alexander J, Bressler SB, Maguire MG, Hawkins BS, Fine SL. Subfoveal neovascular lesions in age-related macular degeneration: guidelines for evaluation and treatment in the macular photocoagulation study. Arch Ophthalmol 1991; 109: 1242-57.

6 Macular Photocoagulation Study Group. Recurrent choroidal neovascularization after argon laser photocoagulation for neovascular maculopathy Arch Ophthalmol 1986; 104: 503-12.

7 Reed MWR, Miller FN, Wieman TJ, Tseng MT, Pietsch CG. The effect of photodynamic therapy on the microCG. The effect of photodynamic therapy
circulation. $₹$ Surg Res $1988 ; 45: 452-9$.

8 Miller H, Miller B. Photodynamic therapy of subretinal neovascularization in the monkey eye. Arch Ophthalmol 1993; 111: 855-60.

9 Kliman GH, Puliafito CA, Stern D, Borirakchanyavat S, Gregory WA. Phthalocyanine photodynamic therapy: new strategy for closure of choroidal neovascularization. Laser Surg Med 1994; 15: 2-10.

10 Kliman GH, Puliafito CA, Grossman GA, Gregory WA Retinal and choroidal vessel closure using phthalocyanine photodynamic therapy. Lasers Surg Med 1994; 15: 11-8.

11 Zeimer RC, Khoobehi B, Niesman MR, Magin RL. A potential method for local drug and dye delivery in the ocular vasculature. Invest Ophthalmol Vis Sci 1988; 29; 1179-83.

12 Zeimer RC, Khoobehi B, Peyman G, Niesman MR, Magin RL. Feasibility of blood flow measurement by externall controlled dye delivery. Invest Ophthalmol Vis Sci 1989 30: $660-7$.

13 Zeimer RC, Guran T, Shahidi M, Mori MT. Visualization of the retinal microvasculature by targeted dye delivery. Invest Ophthalmol Vis Sci 1990; 31: 1459-65.

14 Guran T, Zeimer RC, Shahidi M, Mori M. Quantitative analysis of retinal hemodynamics using targeted dye delivery. Invest Ophthalmol Vis Sci 1990; 31: 2300-6.

15 Kiryu J, Shahidi M, Mori M, Ogura Y, Asrani S, Zeimer R. Noninvasive visualization of the choriocapillaris and its dynamic filling. Invest Ophthalmol Vis Sci 1994; 35: 3724-31.

16 Ogura Y, Guran T, Shahidi M, Mori M, Zeimer R. Feasibility of targeted drug delivery to selective areas of the retina. Invest Ophthalmol Vis Sci 1991; 32: 2351-6.

17 Panagopoulos JA, Svitra PP, Puliafito CA, Gragoudas ES. Photodynamic therapy for experimental intraocular Photodynamic therapy for experimental intraocular
melanoma using chloroaluminum sulfonated phthalocyanine. Arch Ophthalmol 1989; 107: 886-90.
18 Pallikaris IG, Tslimbaris MK, Iliaki OE, Naoumidi II, Georgiades A, Panagopoulos IA. Effectiveness of corneal neovascularization photothrombosis using phthalocyanine and a diode laser (675 nm). Lasers Surg Med 1993; 13: 197-203.

19 Kreimer-Birnbaum M. Modified porphyrins, chlorins, phthalocyanines, and purpurins: second-generation photosensitizers for photodynamic therapy. Sem Hematol 1989; 26: 157-73.

20 Rosenthal I. Phthalocyanines as photodynamic sensitizers. Photochem Photobiol 1991; 53: 859-70.

21 Roberts WG, Smith KM, McCullough JL, Berns MW. Skin photosensitivity and photodestruction of several potential photodynamic sensitizers. Photochem Photobiol 1989; 49: 431-8.

22 Schmidt S, Wagner U, Oehr P, Krebs D. Klinischer einsatz der photocyanischen therapie bei gynakologischen tumorpatienten-antikorper-vermittelte photodynamische lasertherapie als neues onkologisches behandlungsverfahren. Zen Gyn 1992; 114: 307-11.

23 L'Esperance FA. Tunable organic dye laser. In: Ophthalmic Lasers. 3rd ed. St Louis: Mosby, 1989; Vol 1: 276-9.

24 Bebie H, Fankhauser F, Lotmar W, Roulier A. Theoretical estimate of the temperature within irradiated retinal vessels. Arch Ophthalmol 1974; 52: 13-36.

25 American National Standard for the Safe Use of Lasers, ANSI Z-136.1-1993. Orlando: The Laser Institute of America, 1993.

26 Mayhew E, Ito M, Lazo R. Toxicity of non-drug-containing liposomes for cultured human cells. Exp Cell Res 1987; 171: 195-202.

27 Gabizon A, Peretz T, Sulkes A, Ben-Yosef R, Ben-Baruch $\mathrm{N}$, Catane $\mathrm{R}$, et al. Systemic administration of doxorubicin-containing liposomes in cancer patients: a
phase I study. Eur $\mathcal{f}$ Cancer Clin Oncol 1989; 25: phase I stud

28 Perez-Soler R, Lopez-Berestein G, Lautersztain J, AlBaker S, Francis K, Macias-Kiger D, et al. Phase I clinical and pharmacological study of liposomeentrapped cis-bis-neodecanoato-trans- $R, R-1,2$-diaminocyclohexane platinum (II). Cancer Res 1990; 50: 4254-9.

29 Vosika GJ, Cornelius DA, Gilbert CW, Sadlik JR, Bennek JA, Doyle A, et al. Phase I trial of the ImmTher, a new liposome-incorporated lipophilic disaccharide tripeptide. fiposome-incorporated lipophilic

30 Rahman A, Treat J, Roh J, Potkul LA, Alvord WG, Forst D, et al. A phase I clinical trial and pharmacokinetic evaluation of liposome-encapsulated doxorubicin. $\mathcal{F}$ Clin Oncol 1990; 8: 1093-100. 31 Gregoriadis G. Liposome technology: interactions of liposomes
with the biological milieu. 2nd ed. Boca Raton, FL: CRC Press, 1993: Vol 3.

32 Gass JDM. Diseases causing choroidal exudative and hemorrhagic localized detachment of the retina and pigment epithelium. In: Klein EA, ed. Stereoscopic atlas of macular diseases diagnosis and treatment. 3rd ed. St Louis: Mosby, 1987: 68-72. 